\title{
Produção e qualidade do grão do arroz irrigado infestado por adultos de percevejo-das-panículas
}

\author{
Evane Ferreira(1) $^{(1)}$ José Alexandre Freitas Barrigossi(2)
}

(1)In memoriam (2)Embrapa Arroz e Feijão, Rod. Goiânia a Nova Veneza, Km 12, Zona Rural, Caixa Postal 179, CEP $75375-000$ Santo Antônio de Goiás, GO. E-mail: alex@cnpaf.embrapa.br

Resumo - O objetivo deste trabalho foi comparar os danos qualitativos e quantitativos, causados por adultos de Oebalus poecilus (Dallas) Stal, em panículas de 39 genótipos de arroz irrigado. Em campo, as panículas emergentes foram isoladas em gaiolas e infestadas com dois insetos, no início da fase leitosa das espiguetas. Panículas não infestadas serviram de testemunha. Após a colheita, amostras compostas de 50 espiguetas de cada grau de infestação, para cada genótipo, foram semeadas em telado, e a emergência foi determinada após 16 dias. Manchas no grão foram avaliadas em amostras de 50 espiguetas. O percevejo provocou alterações significativas, pois reduziu a massa e o número de espiguetas por panícula, a porcentagem de plântulas emergidas, e aumentou a porcentagem de espiguetas vazias e com grão manchado. A combinação da porcentagem de perda de massa com a de sementes inviáveis foi de 44\%, e da perda de massa com a de espiguetas manchadas, de 81,4\%. A maior porcentagem de plântulas emergidas de espiguetas manchadas ocorreu nos genótipos de ciclo médio. Os genótipos de ciclo curto CNAi 8859, CNAi 8879, CNAi 8885 e CNAi 8886, e os de ciclo médio CNAi 9089, CNAi 9097, CNAi 9150, CNAi 9687 CNAi 9730, CNAi 9747 e CNAi 9778 são os mais tolerantes ao percevejo-daspanículas.

Temos para indexação: Oryza sativa, Oebalus poecilus, Hemiptera, Pentatomidae, genótipos precoces, genótipos tardios.

\section{Yield and grain quality of flood rice infested with adults of rice stink bug}

\begin{abstract}
The objective of this work was to compare the effects of infestation with adults of rice stink bug, Oebalus poecilus (Dallas) Stal, on panicles of 39 genotypes of irrigated rice. In the field, newly emerged panicles were isolated in cages and infested with two insects in the beginning of the milky stage. Non-infested panicles comprised the controls. After harvest, samples of 50 spikelets from each infestation level were seeded in screen house, and the emergence was determined 16 days after planting. Stains in the grain were evaluated in samples of 50 spikelets. The stink bug produced significant alterations, reducing weight and number of spikelets per panicle, and percentage of seedlings, and increasing the percentage of empty spikelets and spikelets with stained grain. Combined percentage of weight loss with no viable seeds was $44 \%$, and combined percentage of weight loss with stained spikelets was $81.4 \%$. The highest percentage of seedlings emerged from stained spikelets was observed in genotypes of median season. The short season genotypes CNAi 8859, CNAi 8879, CNAi 8885 and CNAi 8886, and the median season genotypes CNAi 9089, CNAi 9097, CNAi 9150 CNAi 9687, CNAi 9730, CNAi 9747 and CNAi 9778 are the most tolerant to rice stink bug.
\end{abstract}

Index terms: Oryza sativa, Oebalus poecilus, Hemiptera, Pentatomidae, early genotypes, late genotypes.

\section{Introdução}

O percevejo Oebalus poecilus (Dallas) Stal, 1862 (Heteroptera: Pentatomidae), praga importante do arroz de terras baixas, está distribuído por todo o continente americano, com ocorrências registradas no Brasil desde 1918 (Ferreira et al., 2001). Apresenta grande variação na coloração e nos desenhos do corpo; machos apresentam tamanho menor que fêmeas. O ciclo biológico é reduzido com o aumento da temperatura, e os dias curtos, com mais de 10,5 horas de escuro, induzem diapausa (Albuquerque, 1993). Esse percevejo passa o período de entressafra na forma adulta sexualmente imatura, em diferentes sítios de repouso. Possui vários hospedeiros alternativos, nos quais, durante a primavera, se alimenta, acasala e permanece até a 
fase de emissão de panículas nos arrozais, para onde migram em enxame. Dispersam-se durante o dia, concentram-se novamente à tarde, e milhares de fêmeas podem escolher algumas plantas de arroz para ovipositar, dando origem à conhecida postura de enxame. No amanhecer e no período mais quente do dia, ficam parados e abrigados entre as folhas e hastes.

Nas panículas, o inseto suga as ramificações e as espiguetas, o que resulta em reduções na massa, poder germinativo e padrão comercial. A natureza e extensão do dano dependem do estádio de desenvolvimento das espiguetas e da densidade da infestação. Espiguetas com grão líquido e em massa podem ficar totalmente vazias ou originar grãos atrofiados, com pontos escuros nas glumas, nos sítios de alimentação. $\mathrm{O}$ ataque nos estádios subseqüentes causam áreas escuras na casca e brancas no grão, que também podem escurecer, quando infectadas por fungo. Os grãos ficam enfraquecidos nas regiões danificadas e geralmente se quebram durante o beneficiamento. Quando isto não ocorre, aparecem no arroz descascado, desvalorizando-o.

Considerado como praga principal aguda em lavouras de arroz de vários estados do Brasil, O. poecilus às vezes ocorre simultaneamente com $O$. ypsilongriseus. No Rio Grande do Sul, a introdução de cultivares de arroz de grãos finos e longos, com ciclos diferentes daqueles tradicionais, é responsabilizada pela antecipação da ocorrência do percevejo $O$. poecilus nos arrozais (Martins et al., 2004). Para o seu manejo, são recomendadas práticas culturais, e a promissora para a Região Sul é retardar o plantio de cultivares tolerantes a dias curtos. O objetivo desta prática de manejo é induzir a diapausa no inseto, causada pelas condições climáticas, na fase de florescimento do arroz (Albuquerque, 1993).

Apesar de as cultivares resistentes ao percevejodo-grão ainda não serem utilizadas, os trabalhos que avaliaram a suscetibilidade de genótipos a perdas quantitativas (Ferreira et al., 2002a, 2002b; Ferreira \& Barrigossi, 2004), qualitativas (Chaves et al., 2001) e aos dois tipos de perdas simultaneamente (Ferreira et al., 2002b; Silva et al., 2002) indicam essa possibilidade. O controle biológico depende da preservação dos inimigos naturais conhecidos. O controle químico é geralmente considerado ineficiente, porque O. poecilus movimenta-se continuamente, dentro e entre campos de arroz (Albuquerque, 1993). Inseticidas têm sido utilizados sem que seja considerada a viabilidade econômica (Martins et al., 2004), embora os procedimentos adequados para tomada de decisão já tenham evoluído, conforme descrito por Barrigossi et al. (2002) e Ferreira \& Barrigossi (2004).

O objetivo deste trabalho foi comparar os danos qualitativos e quantitativos, em panículas de 39 genótipos de arroz irrigado, provocados por infestação artificial de adultos de $O$. poecilus.

\section{Material e Métodos}

A comparação de genótipos de arroz irrigado, considerando-se os diferentes tipos de perdas provocadas por O. poecilus, foi realizada com base na metodologia utilizada por Ferreira et al. (2002b). Foram utilizados os genótipos de ciclo curto CNAi 8859, CNAi 8860, CNAi 8870, CNAi 8879, CNAi 8880, CNAi 8886, CNAi 8885, CNAi 9867, CNAi 9834, CNAi 9838, CNAi 9842, CNAi 9853, CNAi 9865, BRS Pelota, IRGA 417, BR Irga 409, Taim, Javaé e SCS BRS111 e os de ciclo médio Jaburu, Biguá, CNAi 8569, CNAi 9018, CNAi 9025, CNAi 9089, CNAi 9090, CNAi 9097, CNAi 9150, CNAi 9687, CNAi 9705, CNAi 9747, CNAi 9748, CNAi 9778, CNAi 9730 CNAi 10390, CNAi 10393, Cica 8, Formoso e Metica 1, integrantes do ensaio de Valor de Cultivo e Uso 2002/2003, para a região tropical.

O experimento foi conduzido em duas etapas, por transplantio de mudas produzidas em telado, na Fazenda Palmital, da Embrapa Arroz e Feijão, em Goianira, GO. Na primeira etapa, os genótipos de ciclo curto foram semeados em bandejas, no dia 20/11/2002, e transplantados para o campo em 16/12/2002. Na segunda etapa, os genótipos de ciclo médio foram semeados em bandejas, em 2/12/2002, e transplantados para o campo em 23/12/2002. Este procedimento foi adotado para facilitar a condução do experimento, principalmente durante as infestações. Utilizou-se o delineamento de blocos ao acaso, com parcelas divididas e cinco repetições. As parcelas foram constituídas de uma fileira de plantas de $5 \mathrm{~m}$, de cada genótipo, espaçadas de $0,8 \mathrm{~m}$. A adubação consistiu de $400 \mathrm{~kg} \mathrm{ha}^{-1}$ do formulado 4-30-16, aplicado a lanço por ocasião dos transplantios, e $150 \mathrm{~kg} \mathrm{ha}^{-1}$ de sulfato de amônio, em cobertura, aos 25 dias após o transplantio.

Durante a florescência, seis panículas de cada parcela foram isoladas em gaiolas confeccionadas de garrafas de plástico transparente, com $2 \mathrm{~L}$ de capacidade, 
sem fundo, com três furos de 0,02 $\mathrm{m}$ de diâmetro em sua maior superfície, embutidas numa manga de tela de náilon de 0,09 m de diâmetro e 0,50 m de comprimento. Depois do isolamento, as panículas foram mantidas em posição vertical, por meio de cordéis amarrados nas extremidades superiores das gaiolas, e por meio de fios de arame liso estendidos sobre o experimento.

No início da fase leitosa das espiguetas, quatro dessas panículas foram infestadas, cada uma com dois adultos de O. poecilus oriundos de criação sustentada na linhagem de arroz CNA 8502, em telado. As outras duas panículas isoladas não foram infestadas e serviram de testemunha. As infestações foram mantidas até a completa maturação das espiguetas e foram inspecionadas em intervalos de cinco ou seis dias para repor percevejos mortos e eliminar massas de ovos.

As subparcelas foram colhidas, cortando-se os colmos logo abaixo da extremidade inferior das gaiolas, e levadas para o laboratório. As espiguetas das panículas sem infestação (SI) e com infestação (CI) foram colhidas, secadas, pesadas, mecanicamente separadas nas categorias vazias e com grão, e contadas. Com esses dados, foram calculados os seguintes valores médios para os tratamentos SI e CI: massa e número de espiguetas por panícula, massa por espigueta, porcentagem de espiguetas vazias e porcentagem de perda de massa por espigueta (PME), pela seguinte equação:

$\mathrm{PME}=[(\mathrm{MSI}-\mathrm{MCI}) / \mathrm{MSI}] 100$,

em que MSI é a massa de espiguetas sem infestação e MCI é a massa de espiguetas com infestação. Em etapas posteriores, amostras de 50 espiguetas com grão, dos tratamentos SI e CI, foram retiradas para avaliação da germinação e mancha no grão.

Para avaliação do poder germinativo, cinco repetições das amostras de 50 espiguetas foram semeadas em caixas de água de $1,3 \times 2,1 \times 0,7 \mathrm{~m}$, com terra homogeneizada, em condições de telado. Considerouse para avaliação o número de plântulas emergidas de espiguetas SI e CI, 16 dias após a semeadura. A porcentagem de sementes viáveis foi determinada multiplicando-se por dois o número de plântulas emergidas de SI e CI, e a porcentagem de perda de emergência de plântulas ou de sementes mortas (REP) foi determinada pela diferença entre a porcentagem de germinação nos tratamentos SI e CI.

Para contagem de manchas no grão, as amostras de espiguetas foram colocadas em tubos de ensaio com solução de hidróxido de sódio a $10 \%$, e submetidas a $70^{\circ} \mathrm{C}$ por dez minutos; foram depois lavadas, examinadas sob fina camada de água, em bandeja de fundo branco (Raí, 1974), e classificadas pela seguinte escala: nota 0 - sem mancha -, e notas 1, 2, 3, e 4 - com manchas que ocupam até 25, 50, 75 e 100\% do grão, respectivamente. Com esses dados, calculou-se um índice de intensidade de manchas (IME) associadas às picadas do percevejo, com variação de 0,1 (mancha mínima) a 20 (grãos totalmente escuros), por meio da equação:

$\mathrm{IME}=\left(0,25 \mathrm{X}_{1}+0,5 \mathrm{X}_{2}+0,75 \mathrm{X}_{3}+\mathrm{X}_{4}\right) /(0,25+0,5+0,75+1,00)$, em que $\mathrm{X}_{1}, \mathrm{X}_{2}, \mathrm{X}_{3}$ e $\mathrm{X}_{4}$ correspondem ao número de espiguetas manchadas na escala de notas. Obteve-se a porcentagem de espiguetas sem manchas nos grãos em SI e CI, multiplicando-se o número de espiguetas de nota 0 por dois. A porcentagem de espiguetas manchadas (PEM) foi obtida pela diferença entre o total e a porcentagem de espiguetas sem manchas.

Os efeitos quantitativos e qualitativos da alimentação do percevejo foram estimados e reunidos com base na metodologia de Ferreira et al. (2002a), em dois tipos de perda: porcentagem de perda de valor como semente PVS $=[\mathrm{PME}+(100-\mathrm{PME}) \mathrm{REP}] / 100$ e porcentagem total de perda PTP $=[\mathrm{PME}+(100$ - PME $) \mathrm{REP}] / 100$.

As análises estatísticas foram realizadas considerando-se os genótipos e graus de infestação como fatores e, também, pelas estimativas das perdas por meio de regressão linear simples entre as médias dos genótipos (SAS Institute, 1990). Comparações das médias gerais dos genótipos, nos graus de infestação, foram feitas pelo teste F; dos graus de infestação em cada genótipo, pelo teste de Tukey; e de mais de duas médias, pelo método de Scott \& Knott (1974), todos a $5 \%$ de probabilidade.

\section{Resultados e Discussão}

Os resultados das análises permitiram constatar, por meio das médias dos tratamentos SI e CI, tanto nos genótipos de ciclo curto quanto nos de ciclo médio, que a infestação com dois adultos de $O$. poecilus provocou alterações significativas na massa e no número de espiguetas por panícula (Tabela 1), na massa por espigueta e na porcentagem de espiguetas vazias (Tabela 2), e na porcentagem de espiguetas sem mancha no grão e de plântulas emergidas (Tabela 3). 
Tabela 1. Massa (g) e número de espiguetas por panícula, sem infestação (SI) e infestada (CI) com dois adultos de Oebalus poecilus, em genótipos de arroz irrigado de ciclos curto e médio(1).

\begin{tabular}{|c|c|c|c|c|}
\hline \multirow[t]{2}{*}{ Genótipo } & \multicolumn{2}{|c|}{ Massa de espiguetas } & \multicolumn{2}{|c|}{ Número de espiguetas } \\
\hline & SI & $\mathrm{CI}$ & SI & $\mathrm{CI}$ \\
\hline & \multicolumn{4}{|c|}{ Ciclo curto } \\
\hline CNAi 8859 & $4,587 \mathrm{Aa}$ & $3,471 \mathrm{Ba}$ & $216,2 \mathrm{Aa}$ & $200,1 \mathrm{Aa}$ \\
\hline CNAi 8860 & $3,856 \mathrm{Ab}$ & $2,323 \mathrm{Bb}$ & $201,9 \mathrm{Aa}$ & $177,5 \mathrm{Ab}$ \\
\hline CNAi 8870 & $4,502 \mathrm{Aa}$ & $2,995 \mathrm{Ba}$ & $229,8 \mathrm{Aa}$ & $215,1 \mathrm{Aa}$ \\
\hline CNAi 8879 & $3,715 \mathrm{Ab}$ & $3,130 \mathrm{Aa}$ & $188,6 \mathrm{Ab}$ & $193,6 \mathrm{Aa}$ \\
\hline CNAi 8880 & $3,809 \mathrm{Ab}$ & $2,745 \mathrm{Ba}$ & $195,9 \mathrm{Ab}$ & $204,3 \mathrm{Aa}$ \\
\hline CNAi 8886 & $3,843 \mathrm{Ab}$ & $3,640 \mathrm{Aa}$ & $190,5 \mathrm{Ab}$ & $210,0 \mathrm{Aa}$ \\
\hline CNAi 8885 & $4,068 \mathrm{Ab}$ & $2,925 \mathrm{Ba}$ & $226,3 \mathrm{Aa}$ & $207,4 \mathrm{Aa}$ \\
\hline CNAi 9867 & $4,258 \mathrm{Aa}$ & $2,565 \mathrm{Bb}$ & $232,0 \mathrm{Aa}$ & $195,7 \mathrm{Ba}$ \\
\hline CNAi 9834 & $4,264 \mathrm{Aa}$ & $2,600 \mathrm{Bb}$ & $235,9 \mathrm{Aa}$ & $210,1 \mathrm{Aa}$ \\
\hline CNAi 9838 & $4,540 \mathrm{Aa}$ & $2,842 \mathrm{Ba}$ & $244,6 \mathrm{Aa}$ & $217,1 \mathrm{Aa}$ \\
\hline CNAi 9842 & $4,203 \mathrm{Aa}$ & $2,383 \mathrm{Bb}$ & $226,1 \mathrm{Aa}$ & $204,9 \mathrm{Aa}$ \\
\hline CNAi 9853 & $3,829 \mathrm{Ab}$ & $2,547 \mathrm{Bb}$ & $203,9 \mathrm{Aa}$ & $188,3 \mathrm{Aa}$ \\
\hline CNAi 9865 & $4,037 \mathrm{Ab}$ & $2,590 \mathrm{Bb}$ & $217,8 \mathrm{Aa}$ & $211,6 \mathrm{Aa}$ \\
\hline BRS Pelota & $3,375 \mathrm{Ac}$ & $2,009 \mathrm{Bb}$ & $186,7 \mathrm{Ab}$ & $168,4 \mathrm{Ab}$ \\
\hline Irga 417 & $3,781 \mathrm{Ab}$ & $3,205 \mathrm{Aa}$ & $161,4 \mathrm{Ab}$ & $181,6 \mathrm{Ab}$ \\
\hline BR Irga 409 & $3,265 \mathrm{Ac}$ & $2,229 \mathrm{Bb}$ & $192,8 \mathrm{Ab}$ & $184,5 \mathrm{Ab}$ \\
\hline Taim & $3,501 \mathrm{Ac}$ & $2,634 \mathrm{Bb}$ & $214,0 \mathrm{Aa}$ & $208,6 \mathrm{Aa}$ \\
\hline Javaé & $2,881 \mathrm{Ac}$ & $2,189 \mathrm{Bb}$ & $116,1 \mathrm{Ac}$ & $135,1 \mathrm{Ac}$ \\
\hline SCS BRS 111 & $2,989 \mathrm{Ab}$ & $1,712 \mathrm{Bb}$ & $177,6 \mathrm{Ab}$ & $155,4 \mathrm{Ac}$ \\
\hline \multirow[t]{2}{*}{ Média } & $3,858 \mathrm{~A}$ & $2,670 \mathrm{~B}$ & $203,1 \mathrm{~A}$ & 193,1B \\
\hline & \multicolumn{4}{|c|}{ Ciclo médio } \\
\hline Jaburu & $4,843 \mathrm{Aa}$ & $2,886 \mathrm{Ba}$ & $196,7 \mathrm{Aa}$ & $186,9 \mathrm{Aa}$ \\
\hline Biguá & $3,475 \mathrm{Ab}$ & $2,055 \mathrm{Bb}$ & $183,0 \mathrm{Ab}$ & $160,3 \mathrm{Ab}$ \\
\hline CNAi 8569 & 4,464Aa & $3,256 \mathrm{Ba}$ & $205,4 \mathrm{Aa}$ & $198,3 \mathrm{Aa}$ \\
\hline CNAi 9018 & $4,080 \mathrm{Ab}$ & $3,282 \mathrm{Ba}$ & $176,3 \mathrm{Ab}$ & $181,8 \mathrm{Aa}$ \\
\hline CNAi 9025 & $4,207 \mathrm{Aa}$ & $3,124 \mathrm{Ba}$ & $185,2 \mathrm{Ab}$ & $180,2 \mathrm{Aa}$ \\
\hline CNAi 9089 & 4,339Aa & $2,882 \mathrm{Ba}$ & $183,9 \mathrm{Ab}$ & $158,2 \mathrm{Ab}$ \\
\hline CNAi 9090 & $3,999 \mathrm{Ab}$ & $2,965 \mathrm{Ba}$ & $176,2 \mathrm{Ab}$ & $171,8 \mathrm{Ab}$ \\
\hline CNAi 9097 & $3,437 \mathrm{Ab}$ & $3,057 \mathrm{Ba}$ & $152,1 \mathrm{Ab}$ & $169,9 \mathrm{Ab}$ \\
\hline CNAi 9150 & $3,883 \mathrm{Ab}$ & $3,131 \mathrm{Ba}$ & $147,8 \mathrm{Ac}$ & $147,7 \mathrm{Ab}$ \\
\hline CNAi 9687 & $4,074 \mathrm{Ab}$ & $3,429 \mathrm{Ba}$ & $206,6 \mathrm{Aa}$ & $205,3 \mathrm{Aa}$ \\
\hline CNAi 9705 & $4,836 \mathrm{Aa}$ & $3,458 \mathrm{Ba}$ & $229,9 \mathrm{Aa}$ & $197,6 \mathrm{Ba}$ \\
\hline CNAi 9747 & $4,134 \mathrm{Ab}$ & $3,227 \mathrm{Ba}$ & $190,1 \mathrm{Aa}$ & $179,8 \mathrm{Aa}$ \\
\hline CNAi 9748 & 4,476Aa & $3,290 \mathrm{Ba}$ & $198,4 \mathrm{Aa}$ & $183,9 \mathrm{Aa}$ \\
\hline CNAi 9778 & $3,574 \mathrm{Ab}$ & $2,945 \mathrm{Aa}$ & $194,9 \mathrm{Aa}$ & $186,3 \mathrm{Aa}$ \\
\hline CNAi 9730 & $3,546 \mathrm{Ab}$ & $2,501 \mathrm{Bb}$ & $173,1 \mathrm{Ab}$ & $164,8 \mathrm{Ab}$ \\
\hline CNAi 10390 & $3,341 \mathrm{Ab}$ & $1,977 \mathrm{Bb}$ & $175,7 \mathrm{Ab}$ & $155,3 \mathrm{Ab}$ \\
\hline CNAi 10393 & 4,689Aa & $3,458 \mathrm{Ba}$ & $187,3 \mathrm{Ab}$ & $160,6 \mathrm{Bb}$ \\
\hline Cica 8 & $3,710 \mathrm{Ab}$ & $2,066 \mathrm{Bb}$ & $173,5 \mathrm{Ab}$ & $165,6 \mathrm{Ab}$ \\
\hline Formoso & 4,473Aa & $3,034 \mathrm{Ba}$ & $198,1 \mathrm{Aa}$ & $171,7 \mathrm{Bb}$ \\
\hline Metica 1 & $3,959 \mathrm{Ab}$ & $2,851 \mathrm{Ba}$ & $172,1 \mathrm{Ab}$ & $167,7 \mathrm{Ab}$ \\
\hline Média & $4,074 \mathrm{~A}$ & $2,944 \mathrm{~B}$ & $185,3 \mathrm{~A}$ & $174,5 \mathrm{~B}$ \\
\hline
\end{tabular}

(1)Nas colunas, médias com letras minúsculas iguais não diferem entre si pelo teste de Scott-Knott; e na linha, médias seguidas por letras maiúsculas iguais não diferem entre si pelo teste $\mathrm{F}$ ou de Tukey a $5 \%$ de probabilidade.
Tabela 2. Massa (g) por espigueta e porcentagem de espiguetas vazias, de panículas sem infestação (SI) e infestadas (CI) com dois adultos de Oebalus poecilus, em genótipos de arroz irrigado de ciclos curto e médio(1).

\begin{tabular}{|c|c|c|c|c|}
\hline \multirow[t]{2}{*}{ Genótipo } & \multicolumn{2}{|c|}{ Massa por espigueta } & \multicolumn{2}{|c|}{ Espiguetas vazias } \\
\hline & SI & $\mathrm{CI}$ & SI & $\mathrm{CI}$ \\
\hline & \multicolumn{4}{|c|}{ Ciclo curto } \\
\hline CNAi 8859 & $0,021 \mathrm{Ab}$ & $0,017 \mathrm{Ba}$ & $17,6 \mathrm{Bb}$ & $31,7 \mathrm{Ab}$ \\
\hline CNAi 8860 & $0,019 \mathrm{Ab}$ & $0,013 \mathrm{Bb}$ & $28,7 \mathrm{Ba}$ & $48,9 \mathrm{Aa}$ \\
\hline CNAi 8870 & $0,020 \mathrm{Ab}$ & $0,014 \mathrm{Bb}$ & $24,9 \mathrm{Bb}$ & $46,2 \mathrm{Aa}$ \\
\hline CNAi 8879 & $0,021 \mathrm{Ab}$ & $0,016 \mathrm{Ba}$ & $33,4 \mathrm{Aa}$ & $36,5 \mathrm{Ab}$ \\
\hline CNAi 8880 & $0,019 \mathrm{Ab}$ & $0,013 \mathrm{Bb}$ & $26,9 \mathrm{Bb}$ & $46,1 \mathrm{Aa}$ \\
\hline CNAi 8886 & $0,020 \mathrm{Ab}$ & $0,017 \mathrm{Aa}$ & $25,5 \mathrm{Ab}$ & $34,7 \mathrm{Ab}$ \\
\hline CNAi 8885 & $0,018 \mathrm{Ab}$ & $0,014 \mathrm{Bb}$ & $29,3 \mathrm{Aa}$ & $38,5 \mathrm{Ab}$ \\
\hline CNAi 9867 & $0,018 \mathrm{Ab}$ & $0,013 \mathrm{Bb}$ & $21,2 \mathrm{Bb}$ & $45,7 \mathrm{Aa}$ \\
\hline CNAi 9834 & $0,018 \mathrm{Ab}$ & $0,013 \mathrm{Bb}$ & $26,2 \mathrm{Bb}$ & $46,4 \mathrm{Aa}$ \\
\hline CNAi 9838 & $0,019 \mathrm{Ab}$ & $0,013 \mathrm{Bb}$ & $24,0 \mathrm{Bb}$ & 43,9Aa \\
\hline CNAi 9842 & $0,019 \mathrm{Ab}$ & $0,012 \mathrm{Bb}$ & $23,1 \mathrm{Bb}$ & $48,1 \mathrm{Aa}$ \\
\hline CNAi 9853 & $0,019 \mathrm{Ab}$ & $0,014 \mathrm{Bb}$ & $25,4 \mathrm{Bb}$ & $41,3 \mathrm{Aa}$ \\
\hline CNAi 9865 & $0,019 \mathrm{Ab}$ & $0,012 \mathrm{Bb}$ & $24,0 \mathrm{Bb}$ & $44,9 \mathrm{Aa}$ \\
\hline BRS Pelota & $0,018 \mathrm{Ab}$ & $0,012 \mathrm{Bb}$ & $33,7 \mathrm{Ba}$ & $45,4 \mathrm{Aa}$ \\
\hline Irga 417 & $0,023 \mathrm{Aa}$ & $0,018 \mathrm{Ba}$ & $11,3 \mathrm{Bb}$ & $23,8 \mathrm{Ab}$ \\
\hline BR IRGA 409 & $0,017 \mathrm{Ab}$ & $0,012 \mathrm{Bb}$ & $37,8 \mathrm{Ba}$ & $53,7 \mathrm{Aa}$ \\
\hline Taim & $0,017 \mathrm{Ab}$ & $0,013 \mathrm{Bb}$ & $35,0 \mathrm{Ba}$ & $52,9 \mathrm{Aa}$ \\
\hline Javaé & $0,026 \mathrm{Aa}$ & $0,016 \mathrm{Ba}$ & $16,3 \mathrm{Bb}$ & $30,7 \mathrm{Ab}$ \\
\hline SCS BRS 111 & $0,017 \mathrm{Ab}$ & $0,011 \mathrm{Bb}$ & $40,5 \mathrm{Ba}$ & $60,7 \mathrm{Aa}$ \\
\hline \multirow[t]{2}{*}{ Média } & $0,019 \mathrm{~A}$ & $0,014 \mathrm{~B}$ & $26,6 \mathrm{~B}$ & $43,2 \mathrm{~A}$ \\
\hline & \multicolumn{4}{|c|}{ Cíclo médio } \\
\hline Jaburu & $0,025 \mathrm{Aa}$ & $0,015 \mathrm{Bc}$ & $10,5 \mathrm{Bb}$ & $35,0 \mathrm{Ab}$ \\
\hline Biguá & $0,019 \mathrm{Ac}$ & $0,013 \mathrm{Bd}$ & $33,5 \mathrm{Ba}$ & $54,9 \mathrm{Aa}$ \\
\hline CNAi 8569 & $0,022 \mathrm{Ab}$ & $0,016 \mathrm{Bc}$ & $19,7 \mathrm{Ba}$ & $32,5 \mathrm{Ab}$ \\
\hline CNAi 9018 & $0,023 \mathrm{Ab}$ & $0,018 \mathrm{Bb}$ & $15,1 \mathrm{Bb}$ & $25,3 \mathrm{Ac}$ \\
\hline CNAi 9025 & $0,023 \mathrm{Ab}$ & $0,017 \mathrm{Bb}$ & $13,7 \mathrm{Bb}$ & $29,8 \mathrm{Ab}$ \\
\hline CNAi 9089 & $0,024 \mathrm{Aa}$ & $0,018 \mathrm{Bb}$ & $7,2 \mathrm{Bb}$ & $25,6 \mathrm{Ac}$ \\
\hline CNAi 9090 & $0,023 \mathrm{Ab}$ & $0,017 \mathrm{Bb}$ & $8,0 \mathrm{Bb}$ & $23,1 \mathrm{Ac}$ \\
\hline CNAi 9097 & $0,023 \mathrm{Ab}$ & $0,019 \mathrm{Bb}$ & $8,0 \mathrm{Ab}$ & $17,5 \mathrm{Ac}$ \\
\hline CNAi 9150 & $0,026 \mathrm{Aa}$ & $0,021 \mathrm{Ba}$ & $18,2 \mathrm{Aa}$ & $25,8 \mathrm{Ac}$ \\
\hline CNAi 9687 & $0,020 \mathrm{Ac}$ & $0,017 \mathrm{Ab}$ & $21,5 \mathrm{Ba}$ & $33,4 \mathrm{Ab}$ \\
\hline CNAi 9705 & $0,021 \mathrm{Ac}$ & $0,018 \mathrm{Ab}$ & $20,1 \mathrm{Ba}$ & $30,7 \mathrm{Ab}$ \\
\hline CNAi 9747 & $0,022 \mathrm{Ab}$ & $0,018 \mathrm{Bb}$ & $13,4 \mathrm{Bb}$ & $25,7 \mathrm{Ac}$ \\
\hline CNAi 9748 & $0,023 \mathrm{Ab}$ & $0,018 \mathrm{Bb}$ & $14,5 \mathrm{Bb}$ & $24,3 \mathrm{Ac}$ \\
\hline CNAi 9778 & $0,018 \mathrm{Ac}$ & $0,016 \mathrm{Ac}$ & $25,8 \mathrm{Aa}$ & $31,0 \mathrm{Ab}$ \\
\hline CNAi 9730 & $0,021 \mathrm{Ac}$ & $0,015 \mathrm{Bc}$ & $25,1 \mathrm{Aa}$ & $34,1 \mathrm{Ab}$ \\
\hline CNAi 10390 & 0,019Ac & $0,013 \mathrm{Bd}$ & $25,3 \mathrm{Ba}$ & $45,4 \mathrm{Aa}$ \\
\hline CNAi 10393 & $0,025 \mathrm{Aa}$ & $0,021 \mathrm{Ba}$ & $14,5 \mathrm{Bb}$ & $24,3 \mathrm{Ac}$ \\
\hline Cica 8 & $0,021 \mathrm{Ac}$ & $0,013 \mathrm{Bd}$ & $13,0 \mathrm{Bb}$ & $45,6 \mathrm{Aa}$ \\
\hline Formoso & $0,023 \mathrm{Ab}$ & $0,018 \mathrm{Bb}$ & $12,5 \mathrm{Bb}$ & $28,2 \mathrm{Ac}$ \\
\hline Metica 1 & $0,023 \mathrm{Ab}$ & $0,017 \mathrm{Bb}$ & $10,4 \mathrm{Bb}$ & $34,7 \mathrm{Ab}$ \\
\hline Média & $0,022 \mathrm{~A}$ & $0,017 \mathrm{~B}$ & $16,5 \mathrm{~B}$ & $31,3 \mathrm{~A}$ \\
\hline
\end{tabular}

(1)Nas colunas, médias com letras minúsculas iguais não diferem entre si pelo teste de Scott-Knott; e na linha, médias seguidas por letras maiúsculas iguais não diferem entre si pelo teste F ou de Tukey a 5\% de probabilidade. 
Tabela 3. Porcentagem de espiguetas sem manchas e de plântulas emergidas de panículas sem infestação (SI) e infestadas (CI) com dois adultos de Oebalus poecilus, em genótipos de arroz irrigado de ciclos curto e médio(1).

\begin{tabular}{|c|c|c|c|c|}
\hline \multirow[t]{2}{*}{ Genótipo } & \multicolumn{2}{|c|}{ Espiguetas sem mancha } & \multicolumn{2}{|c|}{ Plântulas emergidas } \\
\hline & SI & $\mathrm{CI}$ & SI & $\mathrm{CI}$ \\
\hline & \multicolumn{4}{|c|}{ Ciclo curto } \\
\hline CNAi 8859 & $98,8 \mathrm{Aa}$ & $29,6 \mathrm{Bb}$ & $84,4 \mathrm{Aa}$ & $59,6 \mathrm{Ba}$ \\
\hline CNAi 8860 & $99,6 \mathrm{Aa}$ & $22,8 \mathrm{Bc}$ & $74,4 \mathrm{Aa}$ & $46,0 \mathrm{Bb}$ \\
\hline CNAi 8870 & $98,8 \mathrm{Aa}$ & $23,6 \mathrm{Bc}$ & $61,2 \mathrm{Ab}$ & $29,6 \mathrm{Bc}$ \\
\hline CNAi 8879 & $97,6 \mathrm{Aa}$ & $30,0 \mathrm{Bb}$ & $76,4 \mathrm{Aa}$ & $54,4 \mathrm{Bb}$ \\
\hline CNAi 8880 & $98,8 \mathrm{Aa}$ & $22,8 \mathrm{Bc}$ & $77,6 \mathrm{Aa}$ & $53,2 \mathrm{Bb}$ \\
\hline CNAi 8886 & $98,0 \mathrm{Aa}$ & $34,4 \mathrm{Ba}$ & $64,0 \mathrm{Ab}$ & $53,6 \mathrm{Ab}$ \\
\hline CNAi 8885 & $98,8 \mathrm{Aa}$ & $36,8 \mathrm{Ba}$ & $57,6 \mathrm{Ac}$ & $47,2 \mathrm{Ab}$ \\
\hline CNAi 9867 & $98,8 \mathrm{Aa}$ & $24,4 \mathrm{Bc}$ & $65,6 \mathrm{Ab}$ & $32,0 \mathrm{Bc}$ \\
\hline CNAi 9834 & $97,6 \mathrm{Aa}$ & $24,0 \mathrm{Bc}$ & $80,0 \mathrm{Aa}$ & $50,0 \mathrm{Bb}$ \\
\hline CNAi 9838 & $97,2 \mathrm{Aa}$ & $23,2 \mathrm{Bc}$ & $92,4 \mathrm{Aa}$ & $48,0 \mathrm{Bb}$ \\
\hline CNAi 9842 & $98,4 \mathrm{Aa}$ & $20,8 \mathrm{Bc}$ & $88,4 \mathrm{Aa}$ & $47,6 \mathrm{Bb}$ \\
\hline CNAi 9853 & $99,2 \mathrm{Aa}$ & $25,6 \mathrm{Bc}$ & $82,8 \mathrm{Aa}$ & $46,8 \mathrm{Bb}$ \\
\hline CNAi 9865 & $99,6 \mathrm{Aa}$ & $18,8 \mathrm{Bc}$ & $81,2 \mathrm{Aa}$ & $48,4 \mathrm{Bb}$ \\
\hline BRS Pelota & $99,2 \mathrm{Aa}$ & $10,0 \mathrm{Bd}$ & $84,0 \mathrm{Aa}$ & $39,2 \mathrm{Bc}$ \\
\hline Irga 417 & $99,6 \mathrm{Aa}$ & $20,0 \mathrm{Bc}$ & $87,2 \mathrm{Aa}$ & $67,6 \mathrm{Ba}$ \\
\hline BR IRGA 409 & $97,2 \mathrm{Aa}$ & $16,0 \mathrm{Bd}$ & $50,0 \mathrm{Ac}$ & $33,2 \mathrm{Bc}$ \\
\hline Taim & $98,8 \mathrm{Aa}$ & $25,2 \mathrm{Bc}$ & $66,0 \mathrm{Ab}$ & $46,0 \mathrm{Bb}$ \\
\hline Javaé & $98,4 \mathrm{Aa}$ & $27,2 \mathrm{Bb}$ & $68,8 \mathrm{Ab}$ & $46,4 \mathrm{Bb}$ \\
\hline SCS BRS 111 & $99,6 \mathrm{Aa}$ & $28,0 \mathrm{Bb}$ & $49,2 \mathrm{Ac}$ & $40,0 \mathrm{Ac}$ \\
\hline \multirow[t]{2}{*}{ Média } & $98,6 \mathrm{~A}$ & $24,4 \mathrm{~B}$ & $73,3 \mathrm{~A}$ & $46,8 \mathrm{~B}$ \\
\hline & \multicolumn{4}{|c|}{ Ciclo médio } \\
\hline Jaburu & $98,0 \mathrm{Aa}$ & $22,0 \mathrm{Bb}$ & $91,6 \mathrm{Aa}$ & $53,2 \mathrm{Bd}$ \\
\hline Biguá & $99,6 \mathrm{Aa}$ & $23,6 \mathrm{Bb}$ & $92,0 \mathrm{Aa}$ & $62,0 \mathrm{Bc}$ \\
\hline CNAi 8569 & $98,4 \mathrm{Aa}$ & $19,6 \mathrm{Bc}$ & $96,0 \mathrm{Aa}$ & $64,4 \mathrm{Bc}$ \\
\hline CNAi 9018 & $98,4 \mathrm{Aa}$ & $15,2 \mathrm{Bc}$ & $95,6 \mathrm{Aa}$ & $69,6 \mathrm{Bc}$ \\
\hline CNAi 9025 & $99,2 \mathrm{Aa}$ & $22,0 \mathrm{Bb}$ & $96,4 \mathrm{Aa}$ & $74,8 \mathrm{Bb}$ \\
\hline CNAi 9089 & $99,6 \mathrm{Aa}$ & $27,2 \mathrm{Ba}$ & $95,6 \mathrm{Aa}$ & $76,8 \mathrm{Bb}$ \\
\hline CNAi 9090 & $99,2 \mathrm{Aa}$ & $22,0 \mathrm{Bb}$ & $93,6 \mathrm{Aa}$ & $66,8 \mathrm{Bc}$ \\
\hline CNAi 9097 & $96,4 \mathrm{Aa}$ & $18,4 \mathrm{Bc}$ & $87,2 \mathrm{Ab}$ & $61,6 \mathrm{Bc}$ \\
\hline CNAi 9150 & $96,8 \mathrm{Aa}$ & $26,8 \mathrm{Ba}$ & $40,4 \mathrm{Ae}$ & $31,2 \mathrm{Be}$ \\
\hline CNAi 9687 & $92,0 \mathrm{Aa}$ & $22,4 \mathrm{Bb}$ & $76,0 \mathrm{Ac}$ & $65,6 \mathrm{Bc}$ \\
\hline CNAi 9705 & $99,2 \mathrm{Aa}$ & $21,6 \mathrm{Bb}$ & $93,6 \mathrm{Aa}$ & $84,0 \mathrm{Ba}$ \\
\hline CNAi 9747 & $100,0 \mathrm{Aa}$ & $24,4 \mathrm{Ba}$ & $92,4 \mathrm{Aa}$ & $74,0 \mathrm{Bb}$ \\
\hline CNAi 9748 & $99,2 \mathrm{Aa}$ & $21,2 \mathrm{Bb}$ & $86,8 \mathrm{Ab}$ & $57,6 \mathrm{Bc}$ \\
\hline CNAi 9778 & $99,2 \mathrm{Aa}$ & $24,8 \mathrm{Ba}$ & $86,0 \mathrm{Ab}$ & $60,8 \mathrm{Bc}$ \\
\hline CNAi 9730 & $97,2 \mathrm{Aa}$ & $26,8 \mathrm{Ba}$ & $84,0 \mathrm{Ab}$ & $66,0 \mathrm{Bc}$ \\
\hline CNAi 10390 & $98,8 \mathrm{Aa}$ & $21,2 \mathrm{Bb}$ & $94,4 \mathrm{Aa}$ & $49,6 \mathrm{Bd}$ \\
\hline CNAi 10393 & $97,6 \mathrm{Aa}$ & $16,4 \mathrm{Bc}$ & $65,6 \mathrm{Ad}$ & $51,2 \mathrm{Bd}$ \\
\hline Cica 8 & $95,6 \mathrm{Aa}$ & $18,4 \mathrm{Bc}$ & $76,4 \mathrm{Ac}$ & $61,2 \mathrm{Bc}$ \\
\hline Formoso & $97,6 \mathrm{Aa}$ & $18,8 \mathrm{Bc}$ & $94,0 \mathrm{Aa}$ & $60,4 \mathrm{Bc}$ \\
\hline Metica 1 & $100,0 \mathrm{Aa}$ & $18,8 \mathrm{Bc}$ & $98,0 \mathrm{Aa}$ & $84,0 \mathrm{Bc}$ \\
\hline Média & $98,1 \mathrm{~A}$ & $21,6 \mathrm{~B}$ & $86,8 \mathrm{~A}$ & $62,7 \mathrm{~B}$ \\
\hline
\end{tabular}

(1)Nas colunas, médias com letras minúsculas iguais não diferem entre si pelo teste de Scott-Knott; e na linha, médias seguidas por letras maiúsculas iguais não diferem entre si pelo teste $\mathrm{F}$ ou de Tukey a $5 \%$ de probabilidade.
As reduções nas médias de massa e número de espiguetas das panículas infestadas foram semelhantes nos dois grupos de genótipos, tendo sido, respectivamente, de 1,188 g e 10 espiguetas nos genótipos de ciclo curto, e de $1,130 \mathrm{~g}$ e 10,8 espiguetas nos de ciclo médio (Tabela 1). Ocorreram diferenças significativas nas variáveis inerentes aos genótipos de cada grupo e, estas variáveis refletiram diferenças no comportamento de alguns genótipos, com relação aos graus de infestação.

O percevejo não causou variação significativa na massa de espigueta por panícula nos genótipos de ciclo curto CNAi 8879, CNAi 8886 e Irga 417, porém causou variação significativa no número de espiguetas por panícula em CNAi 9867 (Tabela 1). Fato semelhante observa-se nos genótipos de ciclo médio, em que a massa de espiguetas por panícula de CNAi 9778 não diferiu entre os tratamentos, enquanto o número de espiguetas de CNAi 9705, CNAi 10393 e Formoso com infestação teve redução significativa (Tabela 1). Essas diferenças são dependentes de vários fatores que interferem na fecundação e no desenvolvimento das espiguetas (Fageria, 1984). Entretanto, no presente estudo, consideraram-se como principais a incidência de doenças, o atraso no desenvolvimento das espiguetas, no sentido da extremidade para a base da panícula (Pedroso, 1985), e a adequação dessas para a alimentação de O. poecilus.

O efeito quantitativo da alimentação dos adultos de O. poecilus, nos genótipos de arroz de ciclo curto e de ciclo médio, pode ser deduzido da Tabela 2, na qual a porcentagem de espiguetas vazias representa o principal componente da perda de massa de espiguetas, com $\mathrm{R}^{2}=0,73(\mathrm{p}<0,01)$ para os de ciclo curto, e $\mathrm{R}^{2}=0,632(\mathrm{p}<0,01)$ para os de ciclo médio. O outro componente de perda de massa resultou do número de espiguetas em cada nota de dano do percevejo (Figura 1); as reduções médias nas massas correspondentes às notas de 1 a 4 foram 3,9, 13,3, 24,1, e 40,0\%. Outra conseqüência desses graus de dano é evidenciada com a remoção da casca das espiguetas, pelo aspecto dos grãos (Figura 2).

Tanto os genótipos de ciclo curto como os de ciclo médio foram significativamente influenciados pela infestação do percevejo, tendo diminuído a massa por espigueta e aumentado a porcentagem de espiguetas vazias. A redução de massa por espigueta, nos genótipos de ciclo curto e médio, foi igual a $0,005 \mathrm{~g}$, que correspondeu a $26,3 \%$ nos de ciclo curto e $22,7 \%$ nos de ciclo médio. Essas porcentagens estão um pouco 
abaixo da média obtida por Ferreira et al. (2001), que foi de 29,8\%, em dez genótipos de arroz irrigado com grau de infestação semelhante. A porcentagem de espiguetas vazias foi de 16,6\% nos genótipos de ciclo curto e 14,8\% nos de ciclo médio (Tabela 2). O genótipo CNAi 8886 não diferiu quanto aos graus de infestação, para massa por espigueta e porcentagem de espiguetas vazia, sendo que esta variável também não diferiu nos graus de infestação para os genótipos CNAi 8879 e CNAi 8885 (Tabela 2).

Os genótipos de ciclo curto diferiram entre si em cada grau de infestação. Sem infestação, os genótipos Irga 417 e Javaé produziram massa por espigueta significativamente maior do que a dos demais (Tabela 2); quando infestados, ficaram no grupo de maior massa por espigueta, junto com CNAi 8859, CNAi 8879, CNAi 8886. Na ausência do percevejo, os genótipos CNAi 8860, CNAi 8879, CNAi 8885, BRS Pelota, Br Irga 409, Taim e SCS BRS 111 forneceram porcentagens de espiguetas vazias significativamente maiores que os demais,

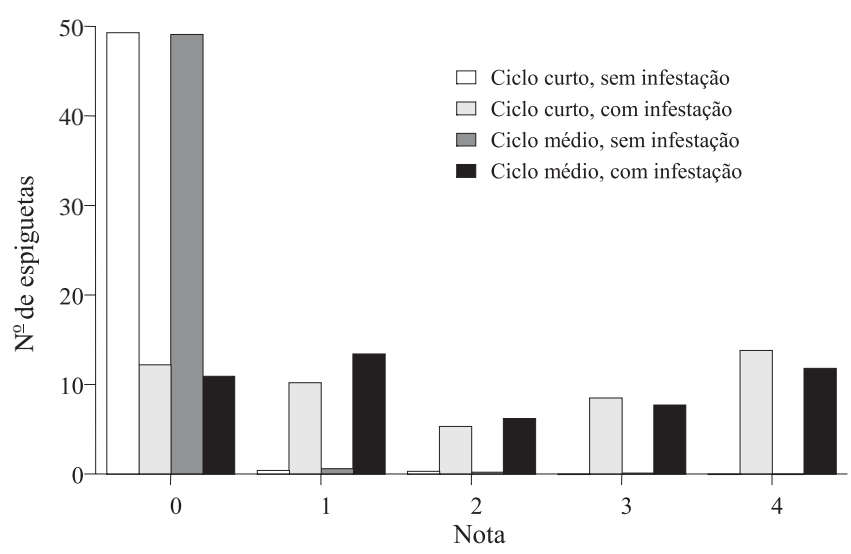

Figura 1. Distribuição das espiguetas de genótipos de arroz irrigado, em uma escala de dano. Nota 0 significa ausência de dano e nota 4 , dano máximo. demonstrando que esta variável também foi influenciada por outros fatores. Sob infestação, esses genótipos mantiveram as maiores porcentagens de espiguetas vazias, exceto CNAi 8879 e CNAi 8885, que passaram a integrar o grupo de menor porcentagem de espiguetas vazias, confirmando que esta variável pode assumir valores mais elevados pela interferência de outros fatores. O fato de os genótipos CNAi 8859, CNAi 8879, CNAi 8886, Irga 417 e Javaé apresentarem maior massa por espigueta e menor porcentagem de espiguetas vazias pode estar ligado à resistência do tipo tolerância ao dano de O. poecilus.

Os genótipos de ciclo médio CNAi 9687, CNAi 9705 e CNAi 9778 não diferiram quanto aos graus de infestação para massa por espigueta, e o CNAi 9778 também não diferiu pela porcentagem de espiguetas vazias, assim como CNAi 9097, CNAi 9150 e CNAi 9730 (Tabela 2).

Os genótipos de ciclo médio diferiram entre si, em cada grau de infestação, pela massa por espigueta e porcentagem de espiguetas vazias. Sem percevejos, os genótipos Jaburu, CNAi 9089, CNAi 9150 e CNAi 10393 produziram massa por espigueta maior que os demais. Com o percevejo, todos os genótipos produziram sementes mais leves, mas CNAi 9150 e CNAi 10393 continuaram com maior massa por espigueta (Tabela 2).

Na ausência do percevejo, o genótipo CNAi 9150 ficou entre os oito com porcentagens de espiguetas vazias significativamente maiores, o que confirma, como no genótipo de ciclo curto, que esta variável pode ser alterada por outros fatores.

Com o percevejo, houve aumento no valor e na variabilidade da porcentagem de espiguetas vazias dos genótipos, originando-se três grupos, com nove genótipos no menos afetado, oito no intermediário e três no mais prejudicado. Os genótipos CNAi 9150 e
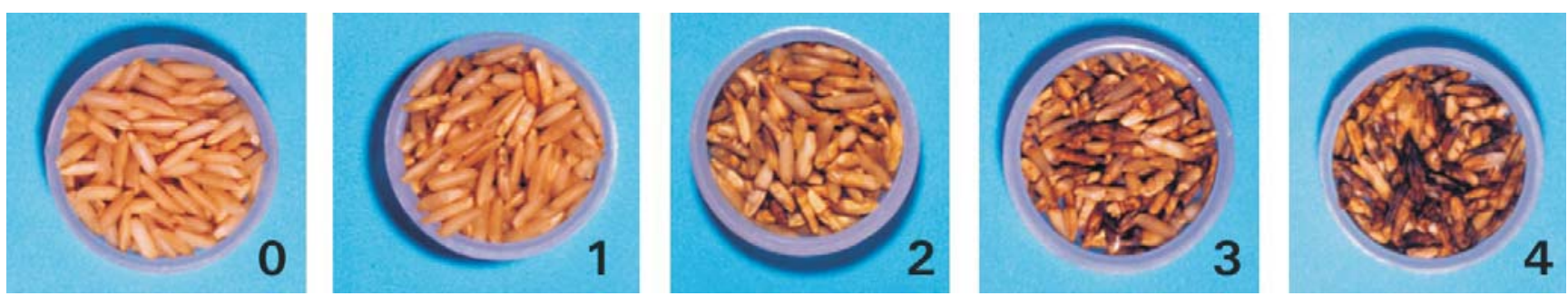

Figura 2. Aspecto dos grãos de arroz, correspondente à escala de notas para avaliar o efeito da alimentação de Oebalus poecilus, em panículas de arroz irrigado. 
CNAi 10393, na presença do percevejo, diferiram dos demais, tendo produzido maiores massas por espigueta e menores porcentagens de espiguetas vazias, o que indica tolerância à alimentação do inseto.

Os efeitos qualitativos dos graus de infestação, nos genótipos de ciclos curtos e médios, foram estudados por meio da porcentagem de espiguetas sem manchas nos grãos e de plântulas emergidas (Tabela 3). Nos genótipos de ciclos curto e médio, respectivamente 25,3 e $11,3 \%$ das espiguetas sem manchas nos grãos não germinaram, enquanto 22,4 e 41,1\% daquelas com grãos manchados germinaram, tendo havido 74,2 e 76,55\% de espiguetas com grãos manchados e reduções de 26,5 e 24,1\% na viabilidade delas ou de plantas emergidas. Isso pode ser explicado pela não coincidência dos sítios de alimentação do inseto com a região do gérmen (Swanson \& Newsom, 1962).

Essas reduções médias, verificadas nas porcentagens de plântulas emergidas, estão um pouco acima daquelas observadas por Chaves et al. (2001), que obtiveram 19,5\% de emergência, 16 dias após a semeadura em telado, de 13 genótipos de arroz irrigado infestados com dois $O$. poecilus adultos por panícula. No entanto, as reduções observadas, neste trabalho, foram inferiores à média de $33 \%$ de germinação de espiguetas provenientes de oito genótipos infestados com a mesma espécie, na proporção de dois por panícula, observados por Ferreira et al. (2002a). Tanto nos genótipos de ciclo curto como nos de ciclo médio (Tabela 3), sempre que o efeito dos graus de infestação foram significativos, houve aumento das porcentagens de grão sem manchas e de plântulas emergidas das espiguetas provenientes de panículas não infestadas.

Na ausência do percevejo, não ocorreu diferença significativa entre os genótipos de ciclo curto e ciclo médio (Tabela 3), na porcentagem de espiguetas sem manchas nos grãos, mas os genótipos de cada grupo diferiram entre si, quando infestados com o percevejo. Os genótipos de cada grupo, infestados ou não, também diferiram pela porcentagem de plantas emergidas.

Na presença do percevejo, os genótipos de ciclo curto CNAi 8885 e CNAi 8886 (Tabela 3) forneceram porcentagens de espiguetas sem manchas nos grãos significativamente maiores que os demais, mas não ficaram entre os de maior porcentagem de plantas emergidas, nem mesmo quando as espiguetas foram desenvolvidas na ausência do percevejo, o que indica que outros fatores interferiram em sua viabilidade.

As maiores porcentagens de espiguetas infestadas sem manchas nos grãos, e o comportamento não dife- renciado dos graus de infestação, para plântulas emergidas, são indicativos de tolerância de CNAi 8885 e CNAi 8886 ao dano de O. poecilus. Os genótipos de ciclo médio CNAi 9089, CNAi 9150, CNAi 9730, CNAi 9747 e CNAi 9778, infestados com o percevejos, diferiram dos demais pelas maiores porcentagens de espiguetas sem manchas nos grãos, mas também não ficaram junto com o CNAi 9705, que apresentou maior porcentagem de plantas emergidas. Sob infestação do percevejo, os genótipos CNAi 9705, CNAi 9025, CNAi 9089 e CNAi 9747 contribuíram com maiores porcentagens de espiguetas sem manchas nos grãos e de plântulas emergidas, e são os mais promissores para reduzir esse tipo de dano.

Estudo de regressão linear simples, entre as porcentagens médias de perda de emergência de plântulas (REP), em função das porcentagens totais de espiguetas manchadas, nas três, duas e uma maiores notas (PEM), e também em função da porcentagem de perda de massa de espiguetas (PME) mostraram que, nos genótipos de ciclo curto, a redução da emergência de plantas foi melhor relacionada com porcentagem total de espiguetas manchadas, representada pelo modelo $\mathrm{REP}=-47,53+0,999$ PEM $\left(\mathrm{R}^{2}=0,297 ; \mathrm{p}<0,01\right)$. Nos genótipos de ciclo médio, a perda de emergência de plântulas foi melhor explicada pela porcentagem de espiguetas manchadas (PEM), calculada sobre as duas maiores notas, representada pelo modelo $\mathrm{REP}=-20,144+1,137 \mathrm{PEM}_{2}\left(\mathrm{R}^{2}=0,356 ; \mathrm{p}<0,01\right)$. Quando se utilizou como variável independente a porcentagem de perda de massa de espigueta (PME), os modelos obtidos foram: $\mathrm{REP}=1,677+0,919 \mathrm{PME}$ $\left(R^{2}=0,264 ; \mathrm{p}<0,05\right)$, para genótipos de ciclo curto, e $\mathrm{REP}=10,686+0,562 \mathrm{PME}\left(\mathrm{R}^{2}=0,166 ; \mathrm{p}<0,05\right)$, para genótipos de ciclo médio.

Diferenças significativas foram observadas, entre as linhagens avaliadas, quanto ao efeito da incidência de $O$. poecilus na redução de massa das espiguetas, redução da emergência de plântulas, aumento de espiguetas manchadas, intensidade de manchas nos grãos, redução na viabilidade de sementes e redução total na produção (Tabela 4). Decidiu-se utilizar como critérios finais, neste trabalho, a redução de massa de espiguetas, a redução do valor como semente e a redução total na produção. A primeira é a mais fácil de se obter e guarda, com as duas últimas, relações lineares significativas que podem ser utilizadas para obtenção de suas estimativas. A redução total na produção tem conotação diferente daquela utilizada por Ferreira et al. 
Tabela 4. Danos relativos à alimentação de dois adultos de Oebalus poecilus por panícula de genótipos de arroz irrigado de ciclos curto e médio, do início da fase leitosa até a completa maturação das espiguetas ${ }^{(1)}$.

\begin{tabular}{|c|c|c|c|c|c|c|}
\hline Genótipo & $\begin{array}{l}\text { RME } \\
(\%)\end{array}$ & $\begin{array}{l}\text { REP } \\
(\%)\end{array}$ & $\begin{array}{c}\text { AEM } \\
(\%)\end{array}$ & IME & $\begin{array}{c}\text { RVS } \\
(\%)\end{array}$ & $\begin{array}{r}\text { RTP } \\
(\%)\end{array}$ \\
\hline & \multicolumn{6}{|c|}{ Ciclo curto } \\
\hline CNA & $17,6 a$ & $24,8 \mathrm{c}$ & $69,2 c$ & $9,2 \mathrm{c}$ & $38,8 \mathrm{~b}$ & 14,4 \\
\hline CNA & & $28,4 \mathrm{~b}$ & $76,8 \mathrm{~b}$ & $11,3 b$ & & 3,1 \\
\hline CNA & $8,4 a$ & $31,6 b$ & $75,2 b$ & $10,4 \mathrm{~b}$ & $2,1 \mathrm{a}$ & 2,5 \\
\hline CNA & $9,0 \mathrm{a}$ & $22,0 \mathrm{c}$ & $67,6 \mathrm{c}$ & $8,9 \mathrm{c}$ & $8,7 \mathrm{~b}$ & $74,1 \mathrm{~b}$ \\
\hline $\mathrm{CNA}$ & & $24,4 \mathrm{c}$ & $76,0 \mathrm{~b}$ & $9,8 \mathrm{~b}$ & & $82,9 \mathrm{a}$ \\
\hline $\mathrm{CNA}$ & $7 \mathrm{a}$ & $10,4 d$ & $63,6 \mathrm{~d}$ & $8,1 \mathrm{~d}$ & $2,0 \mathrm{~b}$ & $67,1 \mathrm{~b}$ \\
\hline & & $10,4 d$ & $62,0 \mathrm{~d}$ & $7,1 \mathrm{~d}$ & $8,3 b$ & 69,4 \\
\hline $\mathrm{NA}$ & & $32,8 b$ & $74,4 \mathrm{~b}$ & $10,1 b$ & $1,4 a$ & $82,0 \mathrm{a}$ \\
\hline & & & $74,4 \mathrm{~b}$ & $10,2 b$ & & $81,7 \mathrm{a}$ \\
\hline & & & $74,0 \mathrm{~b}$ & $10,9 b$ & & 81,5 \\
\hline & & & $77,6 \mathrm{~b}$ & $10,3 \mathrm{~b}$ & $6 a$ & 85,7 \\
\hline & & & $73,6 b$ & $10,1 \mathrm{~b}$ & & $80,6 a$ \\
\hline & & & $81,2 \mathrm{a}$ & $10,9 b$ & & 87,5 \\
\hline & & & $8,8 \mathrm{a}$ & $13,3 \mathrm{a}$ & & 92,6 \\
\hline & & & $79,6 \mathrm{a}$ & $10,4 \mathrm{~b}$ & & 34,7 \\
\hline & & $\mathrm{c}$ & $81,2 \mathrm{a}$ & $12,2 \mathrm{a}$ & & 85,9 \\
\hline & & & $3,6 b$ & $10,0 \mathrm{~b}$ & & 78,7 \\
\hline $\mathrm{Ja}$ & & 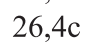 & $1,2 \mathrm{c}$ & & & 80,8 \\
\hline & & $9,2 \mathrm{~d}$ & $71,6 \mathrm{c}$ & $10,3 \mathrm{~b}$ & $0 \mathrm{~b}$ & $80,4 a$ \\
\hline Méd & & & 74,3 & 10,2 & & 80,8 \\
\hline \multirow[t]{2}{*}{$\mathrm{CV}$} & & & 8,5 & 10,8 & & 8,0 \\
\hline & \multicolumn{6}{|c|}{ Ciclo médio } \\
\hline $\mathrm{Ja}$ & $2 a$ & 384 & $76,0 \mathrm{a}$ & $9,9 a$ & $2 a$ & ,9 \\
\hline & & 30 & $75,2 \mathrm{a}$ & $10,0 \mathrm{a}$ & & 82,8 \\
\hline & & & $78,4 \mathrm{a}$ & $10,1 \mathrm{a}$ & & 83,9 \\
\hline & & & & & & 01,0 \\
\hline & & 21 & $77,2 \mathrm{a}$ & $9,0 \mathrm{~b}$ & & 82,2 \\
\hline & & $18,8 \mathrm{c}$ & $74,0 \mathrm{~b}$ & $8,8 \mathrm{~b}$ & & 79,6 \\
\hline & & & $76,8 \mathrm{a}$ & $10,1 \mathrm{a}$ & & 84,3 \\
\hline & & & & & & 79,9 \\
\hline & b & $\mathrm{c}$ & $78,8 \mathrm{a}$ & $9,3 b$ & $1 d$ & 75,3 \\
\hline & & $10,4 \mathrm{c}$ & $70,0 \mathrm{~b}$ & $8,3 \mathrm{~b}$ & & 74,1 \\
\hline & & & $69,6 b$ & $8,7 \mathrm{~b}$ & & 81,6 \\
\hline & & & $77,6 \mathrm{a}$ & $8,1 \mathrm{~b}$ & & 79,5 \\
\hline $\mathrm{CN}$ & $1 \mathrm{~b}$ & $29,2 b$ & $75,6 a$ & $10,1 \mathrm{a}$ & $6 \mathrm{~b}$ & 82,5 \\
\hline & $8 \mathrm{~b}$ & $25,2 b$ & $74,4 \mathrm{~b}$ & $8,9 \mathrm{~b}$ & $35,4 \mathrm{c}$ & 78,1 \\
\hline & $25,7 b$ & $18,0 \mathrm{c}$ & $70,4 b$ & $8,5 \mathrm{~b}$ & $39,1 \mathrm{c}$ & 77,9 \\
\hline & & & $77,6 \mathrm{a}$ & $10,8 \mathrm{a}$ & & 85,0 \\
\hline CNAi 1 & & & $81,2 \mathrm{a}$ & $10,0 \mathrm{a}$ & & 84,2 \\
\hline & $40,6 a$ & & $77,2 \mathrm{a}$ & $9,8 \mathrm{a}$ & $49,3 b$ & 86,4 \\
\hline & $21,7 \mathrm{~b}$ & $33,6 b$ & $78,8 \mathrm{a}$ & $9,8 \mathrm{a}$ & $47,8 b$ & 83,4 \\
\hline $\mathrm{Me}$ & & $34,0 \mathrm{~b}$ & $81,2 \mathrm{a}$ & $9,6 \mathrm{a}$ & $51,0 \mathrm{~b}$ & $85,9 \mathrm{a}$ \\
\hline Média & & & 76,4 & 9,5 & 41,3 & 81,9 \\
\hline $\mathrm{CV}$ & 53,8 & 31,6 & 6,7 & 11,2 & 25,0 & 6,3 \\
\hline
\end{tabular}

(1)RME: redução na massa de espiguetas; REP: redução na emergência de plântulas; AEM: aumento de espiguetas manchadas; IME: intensidade de manchas no grão (0,1 = mínima, 10 = média e 20 = máxima intensidade); RVS: redução na viabilidade das sementes; RTP: redução total na produção. (2)Nas colunas, médias seguidas de letras iguais não diferem entre si pelo teste de Scott-Knott a 5\% de probabilidade. (2002a), que consideraram como perda total aquela que, no caso do presente trabalho, é considerada perda de valor como semente (PVS). Os modelos estabelecidos foram: para genótipos de ciclos curtos, PVS $=8,041+1,419$ PME $\left(R^{2}=0,642 ; \mathrm{p}<0,01\right)$ e PTP $=59,667+0,778 \mathrm{PME}\left(\mathrm{R}^{2}=0,667 ; \mathrm{p}<0,01\right)$; para os genótipos de ciclos médios, PVS $=19,051+0,898 \mathrm{PME}$ $\left(\mathrm{R}^{2}=0,343 ; \mathrm{p}<0,01\right)$ e $\mathrm{PTP}=75,750+0,260 \mathrm{PME}$ $\left(\mathrm{R}^{2}=0,296 ; \mathrm{p}<0,01\right)$.

Os genótipos de ciclos curtos CNAi 8859, CNAi 8885, CNAi 8886 e CNAi 8879 foram os mais tolerantes ao percevejo das panículas, com perda média total de 71,3\%, contra 83,4\% dos restantes suscetíveis. Entre os genótipos de ciclo médio, os menos danificados pelo percevejo O. poecilus foram CNAi 9089, CNAi 9097, CNAi 9150, CNAi 9687, CNAi 9730, CNAi 9747 e CNAi 9778, com perda média total de $77,8 \%$, sendo $84,2 \%$ a perda média total dos suscetíveis.

A proporção de espiguetas com grãos manchados pelo percevejo que germina normalmente deve ser considerada nas avaliações de qualidade das sementes. Os genótipos de arroz irrigado de ciclos curto e médio que tiveram perdas totais significativamente mais baixas, devem ser submetidos a novas avaliações, tendo-se em vista a confirmação de resultados e definição de padrões de resistência.

\section{Conclusões}

1. A infestação de dois adultos de Oebalus poecilus por panícula de arroz irrigado, durante a fase de maturação, causa prejuízos totais, quantitativos e qualitativos em torno de $80 \%$.

2. O dano provocado por dois adultos de Oebalus poecilus, ao longo da fase de maturação das panículas de arroz irrigado, pode ser reduzido, controlando-se o inseto no início dessa fase.

3. Os genótipos de arroz irrigado de ciclos curto e médio apresentam reações diferenciadas aos tipos de perdas, principalmente às associadas com manchas nos grãos.

\section{Referências}

ALBUQUERQUE, G.S. Planting time as a tactic to manage the small rice stink bug, Oebalus poecilus (Hemiptera, Pentatomidae), in Rio Grande do Sul, Brazil. Crop Protection, v.12, p.627630, 1993. 
BARRIGOSSI, J.A.F.; FERREIRA, E.; SANTOS, A.B. dos. Distribuição e amostragem seqüencial de Oryzophagus oryzae (Costa Lima) em lavouras de arroz irrigado do Centro-Oeste do Brasil. In: CONGRESSO DA CADEIA PRODUTIVA DE ARROZ, 1.; REUNIÃO NACIONAL DE PESQUISA DE ARROZ, 7., 2002, Florianópolis. Anais. Santo Antônio de Goiás: Embrapa Arroz e Feijão, 2002. p.451-454. (Embrapa Arroz e Feijão. Documentos, 134).

CHAVES, G.S.; FERREIRA, E.; GARCIA, A.H. Influência da alimentação de Oebalus poecilus (Heteroptera: Pentatomidae) na emergência de plântulas em genótipos de arroz (Oryza sativa) irrigado. Pesquisa Agropecuária Tropical, v.31, p.79-85, 2001.

FAGERIA, N.K. Adubação e nutrição mineral da cultura do arroz. Rio de Janeiro: Campus, 1984. 341p.

FERREIRA, E.; BARRIGOSSI, J.A.F. Reconhecimento e controle dos principais percevejos Oebalus spp. que atacam as panículas de arroz. Santo Antônio de Goiás: Embrapa Arroz e Feijão, 2004. 6p. (Embrapa Arroz e Feijão. Comunicado técnico, 76).

FERREIRA, E.; BARRIGOSSI, J.A.F.; VIEIRA, N.R. de A. Percevejo das panículas do arroz: fauna heteroptera associada ao arroz. Santo Antônio de Goiás: Embrapa Arroz e Feijão, 2001. (Embrapa Arroz e Feijão. Circular técnica, 43). Disponível em: <http:/ /www.cnpaf.embrapa.br/publicacao/circulartecnica/ct_43/ index.htm>. Acesso em: 10 jun. 2005.

FERREIRA, E.; VIEIRA, N.R.A.; BARRIGOSSI, J.A.F.; RANGEL, P.H.N. Perdas quantitativas provocadas por Oebalus poecilus (Dallas, 1851) (Heteroptera: Pentatomidae) nos genótipos do ensaio de arroz irrigado tropical, VCU 2001. In: CONGRESSO DA CADEIA PRODUTIVA DE ARROZ, 1.; REUNIÃO NACIONAL DE PESQUISA DE ARROZ, 7., 2002,
Florianópolis. Anais. Santo Antônio de Goiás: Embrapa Arroz e Feijão, 2002a. p.496-498. (Embrapa Arroz e Feijão. Documentos, 134).

FERREIRA, E.; VIEIRA, N.R. de A.; RANGEL, P.H.N. Avaliação dos danos de Oebalus spp. em genótipos de arroz irrigado. Pesquisa Agropecuária Brasileira, v.37, p.763-768, 2002b.

MARTINS, J.F. da S.; GRÜTZMACHER, A.D.; CUNHA, U.S. da. Descrição e manejo integrado de insetos-praga em arroz irrigado. In: GOMES, A. da S.; MAGALHÃES JÚNIOR, A. de M. (Ed.). Arroz irrigado no Sul do Brasil. Brasília: Embrapa Informação Tecnológica, 2004. p.635-675.

PEDROSO, B.A. Arroz irrigado: obtenção e manejo de cultivares. Porto Alegre: Sagra, 1985. 175p.

RAI, B.K. Losses caused by the paddy bug and "red rice" in Guyana. Lanham: FAO, 1974. p.82-86. (Plant protection bulletin, 22).

SAS INSTITUTE (Cary, Estados Unidos). SAS insight user's guide: version 6. Cary, 1990. 441p.

SCOTT, A.J.; KNOTT, M. Cluster analysis method for grouping means in analysis of variance. Biometrics, v.30, p.507-512, 1974.

SILVA, D.R. e; FERREIRA, E.; VIEIRA, N.R de A. Avaliação de perdas causadas por Oebalus spp. (Hemiptera: Pentatomidae) em arroz de terras altas. Pesquisa Agropecuária Tropical, v.32, p.3945, 2002.

SWANSON, M.C.; NEWSOM, L.D. Effect of infestation by rice stink bug, Oebalus pugnax, on yield and quality in rice. Journal of Economic Entomology, v.55, p.877-879, 1962. 